\title{
Shape Control in Multivariate Barycentric Rational Interpolation
}

\author{
Hoa Thang Nguyen, Annie Cuyt and Oliver Salazar Celis \\ Departement Wis-Inf, Universiteit Antwerpen \\ Middelheimlaan 1, B-2020 Antwerpen-Wilrijk, Belgium
}

\begin{abstract}
The most stable formula for a rational interpolant for use on a finite interval is the barycentric form [1, 2]. A simple choice of the barycentric weights ensures the absence of (unwanted) poles on the real line [3]. In [4] we indicate that a more refined choice of the weights in barycentric rational interpolation can guarantee comonotonicity and coconvexity of the rational interpolant in addition to a polefree region of interest.

In this presentation we generalize the above to the multivariate case. We use a product-like form of univariate barycentric rational interpolants and indicate how the location of the poles and the shape of the function can be controlled. This functionality is of importance in the construction of mathematical models that need to express a certain trend, such as in probability distributions, economics, population dynamics, tumor growth models etc.
\end{abstract}

Keywords: rational function, multivariate, interpolation, shape control, surface PACS: 65D05, 41A20, 41A29, 41A63

\section{BARYCENTRIC RATIONAL INTERPOLATION}

Given $n+1$ mutually distinct points $x_{0}, \ldots, x_{n}$ and function values $f_{0}, \ldots, f_{n}$, the rational functions

$$
r_{n}(x)=\frac{\sum_{i=0}^{n} f_{i} \frac{w_{i}}{\left(x-x_{i}\right)}}{\sum_{i=0}^{n} \frac{w_{i}}{\left(x-x_{i}\right)}}, \quad w_{i} \neq 0
$$

interpolate the values $f_{i}$ at the points $x_{i}$ for any nonzero weights $w_{i}$, in other words $r_{n}\left(x_{i}\right)=f_{i}$. Hence, with respect to interpolation of the given data, the function $r_{n}(x)$ when represented as in (1), is immune to rounding errors in the computation of the coefficients. If we denote

$$
\begin{aligned}
\ell(x) & =\left(x-x_{0}\right) \cdots\left(x-x_{n}\right) \\
\ell_{i}(x) & =\ell(x) /\left(x-x_{i}\right)
\end{aligned}
$$

then $r_{n}(x)$ can be written as $r_{n}(x)=p_{n}(x) / q_{n}(x)$ with

$$
\begin{aligned}
& p_{n}(x)=\sum_{i=0}^{n} f_{i} w_{i} \ell_{i}(x) \\
& q_{n}(x)=\sum_{i=0}^{n} w_{i} \ell_{i}(x) .
\end{aligned}
$$

Hence it is easy to see that the degree in numerator and denominator of $r_{n}(x)$ is at most $n$. A necessary condition for the barycentric weights to satisfy when $r_{n}(x)$ is polefree in $\left[x_{0}, x_{n}\right]$ is [1]

$$
w_{i} w_{i+1}<0, \quad i=0, \ldots, n-1 .
$$

Making use of Descartes' rule of signs or a Lorentz representation of $q_{n}(x)$ [5] we can make $r_{n}(x)$ polefree on the positive real line or in an interval $[a, b]$ respectively. By balancing the weights $w_{i}$ as in [3] we can make $r_{n}(x)$ polefree on the entire real line. 
A rational function of the form (1) clearly does not deliver the minimal degree solution of the rational interpolation problem. There are $n+1$ additional degrees of freedom in the barycentric weights $w_{0}, \ldots, w_{n}$. One way to make good use of these is by imposing some shape conditions on $r_{n}(x)$ [4]. Another way is to add conditions to obtain the minimal degree solution $[6,1,7]$.

In the next sections we show how the above can be generalized to the multivariate case. We restrict our description to the bivariate case, without any loss of generality.

\section{MULTIVARIATE BARYCENTRIC INTERPOLATION}

Let the points $\left(x_{i}, y_{i}\right), i=0, \ldots, n$ be given in $\mathbb{R}^{2}$ and the function values $f_{i}$ at these points. The points are assumed to be mutually distinct, so for $j \neq i$ we have $x_{j} \neq x_{i}$ or $y_{j} \neq y_{i}$. But the coordinates $x_{i}$ need not be mutually distinct and neither do the coordinates $y_{i}$. However, in order to avoid a maze of notations, we assume for simplicity either that the $x_{i}$ are distinct and likewise for the $y_{i}$ (as in Figure 1), or that we are interpolating a full grid of $n+1=(m+1)^{2}$ datapoints (as in Figure 2). In the more general intermediate situation, where some of the $x_{i}$ or $y_{i}$ coincide, one retains in the formulas below only the distinct coordinates and this in each variable.

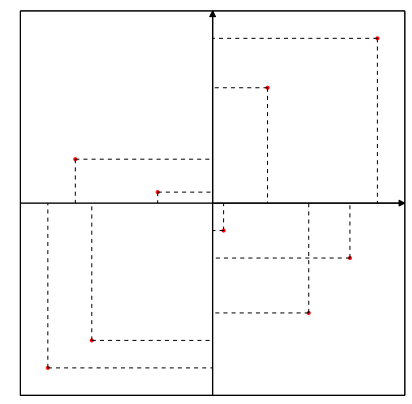

FIGURE 1. Scattered date with $n=8$.

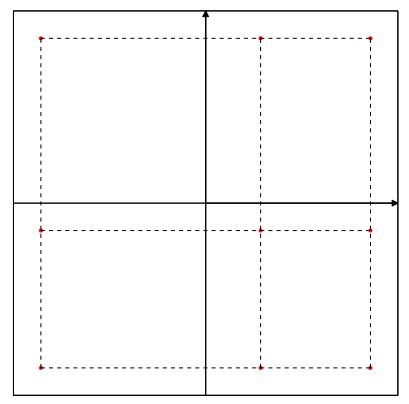

FIGURE 2. Grid data with $m=2$.

The functions

$$
\begin{aligned}
\ell_{j}(x) & =\prod_{i=0, i \neq j}^{n}\left(x-x_{i}\right) \\
\ell_{k}(y) & =\prod_{i=0, i \neq k}^{n}\left(y-y_{i}\right) \\
\ell_{j k}(x, y) & =\ell_{j}(x) \ell_{k}(y)
\end{aligned}
$$

satisfy

$$
\ell_{j k}\left(x_{i}, y_{h}\right)=0, \quad i \neq j \text { or } h \neq k .
$$

The multivariate polynomial

$$
\sum_{i=0}^{n} f_{i} \frac{\ell_{i i}(x, y)}{\ell_{i i}\left(x_{i}, y_{i}\right)}
$$

interpolates the data $f_{i}$ at the points $\left(x_{i}, y_{i}\right)$. The functions $\ell_{i i}(x, y) / \ell_{i i}\left(x_{i}, y_{i}\right)$ in (2) are not the lowest degree polynomials that interpolate zeroes and ones at the $n+1$ datapoints $[8,9]$, but they serve our purpose. More generally, for a grid of $(m+1)^{2}$ function values $f_{j k}$ at points $\left(x_{j}, y_{k}\right)$, the polynomial

$$
\sum_{j, k=0}^{m} f_{j k} \frac{\ell_{j k}(x, y)}{\ell_{j k}\left(x_{j}, y_{k}\right)}
$$

interpolates the data $f_{j k}$. However, as explained, we need not have a grid of function values at our disposal. 
The values $1 / \ell_{i i}\left(x_{i}, y_{i}\right)$ in (2) can be considered as weights in a barycentric formula. It is then easy to see that the multivariate rational function

$$
r_{n}(x, y)=\frac{\sum_{i=0}^{n} f_{i} v_{i} w_{i} \ell_{i i}(x, y)}{\left(\sum_{i=0}^{n} v_{i} \ell_{i}(x)\right)\left(\sum_{i=0}^{n} w_{i} \ell_{i}(y)\right)}
$$

interpolates the data $f_{i}$ at the points $\left(x_{i}, y_{i}\right)$ for whatever nonzero values for the weights $v_{i}$ and $w_{i}$. Again (3) does not constitute the minimal degree multivariate rational interpolant. Additional conditions can be imposed on the barycentric weights $v_{i}$ and $w_{i}$. Also, more generally, the rational function

$$
\frac{\sum_{j, k=0}^{m} f_{j k} v_{j} w_{k} \ell_{j k}(x, y)}{\left(\sum_{i=0}^{n} v_{i} \ell_{i}(x)\right)\left(\sum_{i=0}^{n} w_{i} \ell_{i}(y)\right)}
$$

interpolates a grid of $(m+1)^{2}$ values $f_{j k}$ at points $\left(x_{j}, y_{k}\right)$.

It is easy to choose the weights $v_{i}$ and $w_{i}$ such that the rational function $r_{n}(x, y)$ does not have poles in a specified interval or in $\mathbb{R}^{2}$. The following lemma takes care of that.

Let the orderings $\kappa(i)$ and $\lambda(i)$ of the indices $0, \ldots, n$ be such that $x_{\kappa(0)}<x_{\kappa(1)}<\ldots<x_{\kappa(n)}$ and $y_{\lambda(0)}<y_{\lambda(1)}<$ $\ldots<y_{\lambda(n)}$. In the case of a grid of datapoints $n$ needs to be replaced by $m$.

Lemma 1. Let $v_{\kappa(i)}=(-1)^{\kappa(i)} v_{i}$ and $w_{\lambda(i)}=(-1)^{\lambda(i)} \omega_{i}$ with $v_{i}>0$ and $\omega_{i}>0$. If for $a<x_{\kappa(0)}, b>x_{\kappa(n)}, c<$ $y_{\lambda(0)}, d>y_{\lambda(n)}$ we have

$$
\begin{aligned}
\frac{v_{i-1}}{b-x_{\kappa(i-1)}}<\frac{v_{i}}{b-x_{\kappa(i)}} \quad i=1, \ldots, n \\
\frac{v_{i}}{x_{\kappa(i)}-a}>\frac{v_{i+1}}{x_{\kappa(i+1)}-a}, \quad i=0, \ldots, n-1 \\
\frac{\omega_{i-1}}{d-y_{\lambda(i-1)}}<\frac{\omega_{i}}{d-y_{\lambda(i)}} \quad i=1, \ldots, n \\
\frac{\omega_{i}}{y_{\lambda(i)}-c}>\frac{\omega_{i+1}}{y_{\lambda(i+1)}-c}, \quad i=0, \ldots, n-1
\end{aligned}
$$

then $r_{n}(x, y)$ given by (3) does not have poles in $(a, b) \times(c, d)$. With $a=x_{\kappa(0)}, b=x_{\kappa(n)}, c=y_{\lambda(0)}, d=y_{\lambda(n)}$ the conditions (4) need only be satisfied for $i=1, \ldots, n-1$.

Proof. It is clear that the denominator of $r_{n}(x, y)$ can only have zeroes of the form $x=\alpha$ or $y=\beta$. We show that such zeroes are excluded in the region of interest by proving that the factor in $x$ and that in $y$ in the denominator of (3) are zerofree in $(a, b)$ and $(c, d)$ respectively. To do so we use an idea similar to the one in [3]. Because the $x_{i}$ and the $y_{i}$ are assumed mutually distinct to simplify the notation (otherwise it is just a matter of keeping track which and how many distinct coordinates $x_{i}$ and $y_{i}$ we have), we can denote

$$
\begin{aligned}
\ell(x, y) & =\prod_{i=0}^{n}\left(x-x_{i}\right)\left(y-y_{i}\right) \\
q_{n}(x, y) & =\ell(x, y)\left(\sum_{i=0}^{n} \frac{v_{i}}{x-x_{i}}\right)\left(\sum_{i=0}^{n} \frac{w_{i}}{y-y_{i}}\right) .
\end{aligned}
$$

Numerator and denominator of (3) can be rewritten as

$$
r_{n}(x, y)=\frac{\ell(x, y) \sum_{i=0}^{n} \frac{f_{i} v_{i} w_{i}}{\ell\left(x-x_{i}\right)\left(y-y_{i}\right)}}{\ell(x, y)\left(\sum_{i=0}^{n} \frac{v_{i}}{x-x_{i}}\right)\left(\sum_{i=0}^{n} \frac{w_{i}}{y-y_{i}}\right)}
$$


With the interpolation points $\left(x_{i}, y_{i}\right)$ we associate open intervals $I_{i}=\left(x_{\kappa(i-1)}, x_{\kappa(i)}\right)$ and $H_{i}=\left(y_{\lambda(i-1)}, y_{\lambda(i)}\right)$ where we put $x_{\kappa(-1)}=y_{\lambda(-1)}=-\infty$ and $x_{\kappa(n+1)}=y_{\lambda(n+1)}=+\infty$. Then for $(x, y) \in I_{j} \times H_{k}$ we can write

$$
q_{n}(x, y)=\ell(x, y)(r(x)+s(x))(u(y)+v(y))
$$

where

$$
\begin{aligned}
& r_{i}(x)= \begin{cases}0, & x<x_{\kappa(i)} \\
v_{\kappa(i)} /\left(x-x_{\kappa(i)}\right), & x>x_{\kappa(i)}\end{cases} \\
& s_{i}(x)= \begin{cases}v_{\kappa(i)} /\left(x-x_{\kappa(i)}\right), & x<x_{\kappa(i)} \\
0, & x>x_{\kappa(i)}\end{cases} \\
& r(x)=\sum_{i=0}^{j-1} r_{i}(x), \quad s(x)=\sum_{i=j}^{n} s_{i}(x) .
\end{aligned}
$$

and

$$
\begin{aligned}
& u_{i}(y)= \begin{cases}0, & y<y_{\lambda(i)} \\
w_{\lambda(i)} /\left(y-y_{\lambda(i)}\right), & y>y_{\lambda(i)}\end{cases} \\
& v_{i}(y)= \begin{cases}w_{\lambda(i)} /\left(y-y_{\lambda(i)}\right), & y<y_{\lambda(i)} \\
0, & y>y_{\lambda(i)}\end{cases} \\
& u(y)=\sum_{i=0}^{k-1} u_{i}(y), \quad v(y)=\sum_{i=k}^{n} v_{i}(y) .
\end{aligned}
$$

If the weights $v_{i}$ and $w_{i}$ are such that for $x \in I_{j}, j=1, \ldots, n$ and $y \in H_{k}, k=1, \ldots, n$ we have

$$
\begin{aligned}
\left|r_{i}(x)\right|>\left|r_{i-1}(x)\right|, & & i=1, \ldots, j-1 \\
\left|s_{i}(x)\right|>\left|s_{i+1}(x)\right|, & & i=j, \ldots, n-1, \\
\left|u_{i}(y)\right|>\left|u_{i-1}(y)\right|, & & i=1, \ldots, k-1 \\
\left|v_{i}(y)\right|>\left|v_{i+1}(y)\right|, & & i=k, \ldots, n-1,
\end{aligned}
$$

then the signs of both $r(x)$ and $s(x)$ are that of $(-1)^{\kappa(j-1)}$ and the signs of both $u(y)$ and $v(y)$ are that of $(-1)^{\lambda(k-1)}$. This can be seen by summing $r(x)$ and $u(y)$ from 0 to $j-1$ and $k-1$ respectively and summing $s(x)$ and $v(y)$ from $n$ to $j$ and $k$ respectively. So $r(x)+s(x)$ only changes sign at each $x_{\kappa(i)}$, as does $\ell(x, y)$, and consequently $q_{n}(x, y)$ does not change sign. The same is true for $u(y)+v(y)$. Hence both factors in $q_{n}(x, y)$ are zerofree in $(a, b) \times(c, d)$. Making use of the fact that the $r_{i}(x), u_{i}(y)$ and $s_{i}(x), v_{i}(y)$ are hyperbola and that their vertical asymptotes are ordered since $x_{\kappa(0)}<x_{\kappa(1)}<\ldots<x_{\kappa(n)}$ and $y_{\lambda(0)}<y_{\lambda(1)}<\ldots<y_{\lambda(n)}$, the conditions (5) are satisfied if with $v_{i}=(-1)^{\kappa(i)} v_{i}$ and $w_{i}=(-1)^{\lambda(i)} \omega_{i}$ the conditions (4) are satisfied.

It is obvious that the same property holds for the rational interpolant on a grid of values because its denominator has the same form.

The choice to take both $v_{i}$ and $\omega_{i}$ nonzero also guarantees that $r_{n}(x, y)$ is always defined and finite in $(a, b) \times(c, d)$. The denominator does not have zeroes in $(a, b) \times(c, d)$ and hence numerator and denominator of $r_{n}(x, y)$ cannot have common zeroes in $(a, b) \times(c, d)$. Consequently the occurrence of so-called unattainable interpolation points [10] is impossible.

Let us illustrate the above with some graphs of functions $r_{n}(x, y)$ where the different weights $v_{i}$ and $w_{i}$ satisfy (4). It is clear from these graphical illustrations that there is still an ample choice of interpolants, each displaying a different shape. In the following section we indicate the possibilities for further control of the shape.

Given here are values $f_{k \ell}$ at points $\left(x_{k}, y_{\ell}\right)$ on a $3 \times 3$ grid with $x_{0}=-3, x_{1}=1, x_{2}=3, y_{0}=-3, y_{1}=-0.5, y_{2}=3$. The function values $f_{k \ell}$ (in matrix notation where the row index is $k+1$ and the column index $\ell+1$ ) are

$$
\left(\begin{array}{ccc}
0 & -0.3 & 0 \\
0.2 & 2.5 & -0.2 \\
0 & 0 & 0.3
\end{array}\right) \text {. }
$$


For $v_{i}$ and $w_{i}$ given by $v_{0}=0.4950, v_{1}=-1.000, v_{2}=0.4288, w_{0}=0.4287, w_{1}=-1.000, w_{2}=0.4603$ the rational interpolant is shown in Figure 3. Another look and feel is obtained by choosing $v_{0}=1.000, v_{1}=-0.3402, v_{2}=0.4555$ and $w_{0}=1.000, w_{1}=-5.9536, w_{2}=14.0084$ as in Figure 4.

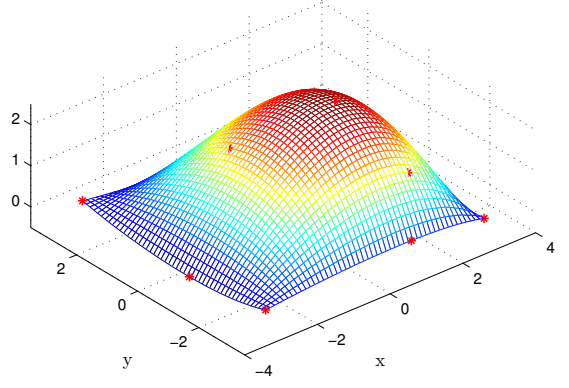

FIGURE 3. $r_{8}(x, y)$.

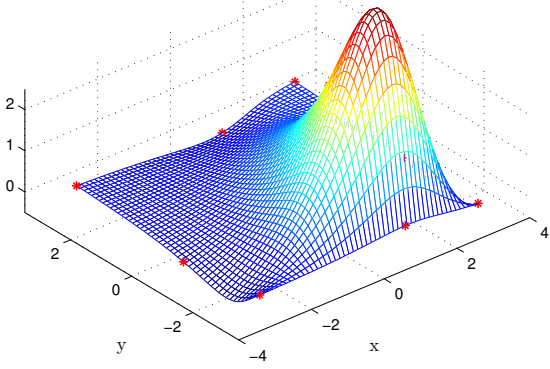

FIGURE 4. $r_{8}(x, y)$.

\section{DERIVATIVES OF THE BARYCENTRIC INTERPOLANT}

The representation (3) takes the form of the univariate barycentric formula (1) by introducing either

$$
F_{i}=r_{n}\left(x_{i}, y\right)=\frac{f_{i} w_{i} \ell_{i}(y)}{\sum_{i=0}^{n} w_{i} \ell_{i}(y)}
$$

or

$$
G_{i}=r_{n}\left(x, y_{i}\right)=\frac{f_{i} v_{i} \ell_{i}(x)}{\sum_{i=0}^{n} v_{i} \ell_{i}(x)} .
$$

The same can be done on a grid of $(m+1)^{2}$ data. Then regarded as a function in $x,(3)$ takes the form

$$
\frac{\sum_{i=0}^{n} F_{i} v_{i} \ell_{i}(x)}{\sum_{i=0}^{n} v_{i} \ell_{i}(x)}
$$

and as a function in $y$,

$$
\frac{\sum_{i=0}^{n} G_{i} w_{i} \ell_{i}(y)}{\sum_{i=0}^{n} w_{i} \ell_{i}(y)} .
$$

Consequently we can use the formulas for the derivatives given in [1] to obtain the partial derivatives

$$
\frac{\partial r_{n}(x, y)}{\partial x}=\left\{\begin{array}{l}
\frac{\sum_{i=0}^{n} \frac{r_{n}(x, y)-r_{n}\left(x_{i}, y\right)}{x-x_{i}} \frac{v_{i}}{x-x_{i}}}{\sum_{i=0}^{n} \frac{v_{i}}{x-x_{i}},} \quad x \neq x_{j}, \quad j=0, \ldots, n \\
-\left(\sum_{i=0, i \neq j}^{n} v_{i} \frac{r_{n}\left(x_{j}, y\right)-r_{n}\left(x_{i}, y\right)}{x-x_{i}}\right) \\
v_{j}, \quad x=x_{j},
\end{array}\right.
$$




$$
\frac{\partial r_{n}(x, y)}{\partial y}=\left\{\begin{array}{l}
\frac{\sum_{i=0}^{n} \frac{r_{n}(x, y)-r_{n}\left(x, y_{i}\right)}{y-y_{i}} \frac{w_{i}}{y-y_{i}}}{\sum_{i=0}^{n} \frac{w_{i}}{y-y_{i}}}, \quad y \neq y_{j}, \quad j=0, \ldots, n \\
\frac{-\left(\sum_{i=0, i \neq j}^{n} w_{i} \frac{r_{n}\left(x, y_{j}\right)-r_{n}\left(x, y_{i}\right)}{y-y_{i}}\right)}{w_{j}}, \quad y=y_{j},
\end{array}\right.
$$

These partial derivatives can be combined into directional derivatives or used for the computation of higher derivatives. Then following the ideas of [4] conditions can be imposed to control the shape of the polefree barycentric rational interpolant. We conclude with the following example.

We take the same data as above. It is easy to see from Figure 4 that for $y=3$ the rational interpolant is not convex. Imagine we want it to be convex along that stretch. Then using the technique from [4] for the partial derivative $\partial r_{8}(x, 3) / \partial x$ we find that the weights $v_{i}$ need to be changed to (for instance) $v_{0}=1, v_{1}=-1.8, v_{2}=1$. We emphasize that the change is computed such that it preserves the guarantee of a polefree interpolant. The result of the change is shown in Figure 5 and in Figure 6 one finds the projection $r_{8}(x, 3)$ before and after the change of weights.

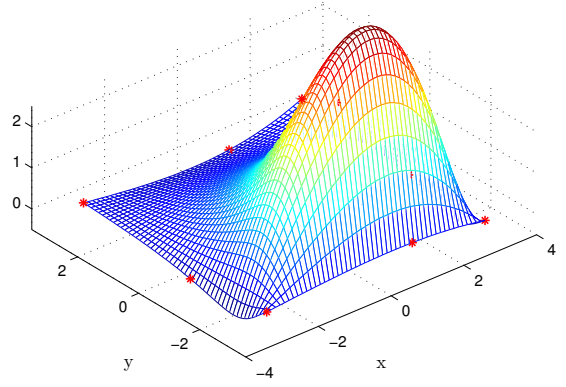

FIGURE 5. Convex $r_{8}(x, y)$.
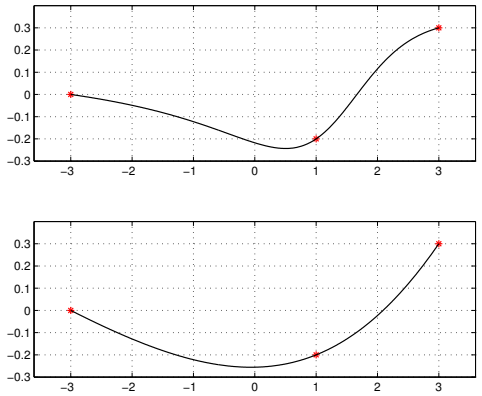

FIGURE 6. Before and after.

\section{REFERENCES}

1. C. Schneider, and W. Werner, Math. Comp. 47, 285-299 (1986).

2. J.-P. Berrut, R. Baltensperger, and H. Mittelmann, "Recent developments in barycentric rational interpolation," in Trends and applications in constructive approximation, edited by M. de Bruin, D. Mache, and J. Szabados, International series of numerical mathematics, Birkhäuser Verlag, Basel, 2005, pp. 27-52.

3. J.-P. Berrut, Comput. Math. Appl. 15, 1-16 (1988).

4. H. T. Nguyen, A. Cuyt, and O. S. Celis, (to appear) (2010).

5. S. N. Bernstein, Soobshch. Kharkov matem. ob-va 14, 227-228 (1915).

6. J.-P. Berrut, and H. Mittelmann, J. Comput. Appl. Math. 78, 355-370 (1997).

7. X. Zhu, and G. Zhu, J. Comput. Appl. Math. 148, 341-348 (2002), ISSN 0377-0427.

8. T. Sauer, Adv. Comput. Math. 3, 219-237 (1995), ISSN 1019-7168.

9. T. Sauer, and Y. Xu, Math. Comp. 64, 1147-1170 (1995).

10. O. Salazar Celis, A. Cuyt, and J. Van Deun, "Symbolic and interval rational interpolation: the problem of unattainable data," in International conference on numerical analysis and applied mathematics, edited by T. Simos, G. Psihoyios, and C. Tsitouras, 2008, vol. 1048 of AIP Conference Proceedings, pp. 466-469. 
Copyright of AIP Conference Proceedings is the property of American Institute of Physics and its content may not be copied or emailed to multiple sites or posted to a listserv without the copyright holder's express written permission. However, users may print, download, or email articles for individual use. 